\title{
Enterprise Network Security Management System Construction Research Based on Cloud Computing Technology
}

\author{
Enfeng Zhou \\ Dongfang College, Shandong University of Finance and Economics, Tai'an Shandong, \\ 271000, China
}

Keywords: Cloud computing technology, Enterprise, Network security management system.

\begin{abstract}
With the constant development of economy in our country in recent years, the role of network technology in contemporary society has become more and more prominent. Network has become an indispensable important information tool for modern people's daily life and work, while cloud computing technology as a new kind of computer technology although can provide convenient service for people, at the same time, it also has hidden danger in network security. Therefore, to strengthen the construction of enterprise network security management system under cloud computing technology is particularly important. This article elaborates the important role cloud computing technology plays in the safe operation of enterprise network security management system, exploring the specific role computing technology plays in the construction of enterprise network security management system and putting forward strategies to further perfect enterprise network security management system under computing technology based on the overview of cloud computing.
\end{abstract}

\section{Introduction}

Currently, human has entered the new era of information technology, especially the computer technology represented network technology has obtained rapid development and provided a lot of Internet services, information and other resources, this also makes new technologies such as cloud computing technology etc. enter more and more into people's work and life. Through the use of data computation, storage and application function, all aspects of modern people become more convenient. However, because cloud computing is a new type of technology on the basis of network, although it can provides users with more convenient services and guarantee the data security, still there are some problems in network security. Under this situation, it is necessary to do research on the construction of computer network security management system under the condition of cloud computing to maximize enterprise network system security performance.

\section{Overview of cloud computing}

The so-called cloud computing mainly refers to the calculation way with the modern network information technology as the center, the important role of which is to provide the increase, decrease and delivery etc. of all kinds of service. The author thinks that resources in cloud computing tend to be virtualized and dynamic. The "cloud" of cloud computing can be said to be the kenning of information network. Compared to traditional calculation, cloud computing has great advantages. According to one source, the computing power cloud computing has can reach even 10 trillion times per second. Because cloud computing has extremely strong computing power, the application of which has realistic possibility in multiple aspects, such as weather forecast, stock, and nuclear explosion. 


\section{Important role cloud computing technology plays in the safe operation of the enterprise network management system}

The first is that it can better regulate and control. Compared with other servers, the prominent advantage cloud computing has is centralized supervision and control. Through centralized supervision, it is able to quickly push the supervision and control on enterprise network security system. Obviously, there are still possibilities for cloud computing to encounter attacks. But this is also a problem that any kind of computer system are difficult to avoid. It requires each enterprise users can store their applications and data in cloud. Because cloud is relatively concentrated, bugs and attack measures it queries are also more effective.

The second is it can ensure that the stored user's data can get relatively good protection. The advantages of centralized supervision and control cloud computing has are also reflected in the proper protection of user data of each store enterprise at the same time. Enterprise network users put their own data stored in the cloud completely and protect, backup and restore them properly. Its level is obviously higher than personal PC or other terminals. At the same time, cloud computing has also the function of using Internet from anywhere else to open the applications. In this way, it can effectively avoid the user data copied to other personnel's mobile storage devices, which further effectively avoid the data leakage problems.

The third is that it is able to make a backup of data more convenient. In the interior of the cloud computing system, the replicated process of action of which is relatively quick, the cloud backup data also therefore becomes quicker and more efficient. Enterprise users can use cloud computing and applications to make various special instructions, so they can finish the corresponding replicated action and the replicated action can transmit the user information to the Internet.

The fourth is that it is able to updating the computer system vulnerabilities, patches and so on. Because the application of enterprise network management system often runs in a special environment and can be updated with the development of cloud computing, using cloud computing technology can greatly enhance the safety performance of network management system. Of course, the result is, the security of the application of enterprise computer network system then can get very good ascension naturally.

\section{Specific application of cloud computing in the construction of enterprise network security management system}

As the fast development of modern science and technology especially the application of modern network technology, the development of network security management system the enterprise shall use in the process of management is in a trend of scientific, systematic, large-scale, and high-effectiveness. To ensure that the enterprise network management system can be more safely operated, it requires real-time maintenance in implementation so as to improve the sustainable development of the enterprise Intranet system. To apply the cloud computing technology to the enterprise network system security management, the following points are mainly required:

The first is to promote the application of cloud computing system. Cloud computing is a collection system of multi-layer service usually constructed by four levels, storage layer, base layer, access layer and interface layer. The system means, in the process of enterprise network system security management, network storage and equipment take the storage layer as their base, because the distributed geographical positions are different, cloud physical devices are different. The connection between each other-different geographical positions and cloud physical devices is realized through intranet. The base layer is the application of related systems such as cluster-type and distributed system etc. to make the stored equipment matched in the cloud can work in cooperation. This layer also contains content of confidentiality, data backup, etc. Access layer refers to the application of the basic and high-level network security management system as well as pushing efficient operation of security software based on software development platform. The interface layer is the component part 
most used in enterprise network system security management. Only through the interface layer can the operational data of enterprise internal network system of be real obtained.

The second is to promote the comprehensive integration of information of cloud computing system. This integration is often completed by using cloud computing technology. For example, the information sharing function in the enterprise internal network security management system is to use the public information model to promote the exchange and share of all kinds of data in enterprise internal database. On this basis, it can also use automatic analysis technology to integrate the useful resources originally very decentralized in the system. Unifying all resources in the cloud system through all information points in the system and sending data demand to public information platform in the network system of this enterprise. While it is able to sort and calculate resources in the system, further returning the computing structure to each information point based on the sorting and calculation of effective cloud resources.

The third is to promote the application of key technology of cloud computing system. The first is the data security technology. This can be said to be the key technology of cloud computing system. Through the promotion of application of cloud computing in the enterprise internal network system, the scattered storage methods can ensure absolutely safe of data. In the safe operation of enterprise network system, it should implement comprehensive analysis of relative technologies such as data processing, enterprise user constraints, resource allocation etc. to really make sure the application data is more stable. So, in the operation of cloud computing system, cloud computing must strengthen the application of hidden functions of all kinds of data to ensure all the data are stable to use, which can be protected by using data encryption technique. Of course, cloud computing security technology can ensure the security performance of data for enterprise users who use this system as well as ensure more safe sharing of the users' data. The second is the dynamic task technology. This technology has great value for system. The calculation methods dynamic task technology introduces are only transient, but also static, and thus have the diversity characteristics. As the calculation time is not stable, and the calculation depends on each other, it increases the difficulty of computing tasks. Because of this, in order to ensure safe and efficient operation of enterprise network system, distributed files can be combined with the local files through mutually combination of between task allocation and dynamic allocation pushed by cloud computing. In this way, they can improve the utilization rate of resources, further let the time loss caused by data transmission fall. The last is the integrated data management technology. It should use this technology to push the realization of unified operation of data model and lower the data loss and failures caused by transformations of all kinds of model effectively in the effective sorting of enterprise network security system. EICCIM international standard is mainly used in current data models. Of course, it can also use E format replace it. As for calculation of input data, it can use the merger method of BPA and PSASP.

\section{Strategies for further perfecting enterprise network security management system on the basis of cloud computing technology}

The fist is to strengthen enterprise network security protection responsibility under the cloud computing technology. The promotion of protection responsibility of enterprise network security first should starts from the implementation of identity authentication of net security management system. It is not only the important link to ensure network security, but also the first against defense for preventing hackers from entering in. It is necessary to form a good network awareness and do reasonable protection on network information and related data to be able to avoid unauthorized access to a great extent so as to eliminate all kinds of unnecessary harms. Under general conditions, users only master the basic computer security operation methods can effectively improve the security of enterprise computer network. For example, a user should as much as possible not use related data in public computer, or avoid using the same password in the process of data storage, this is the outstanding performance of promoting the enterprise network security awareness. At the same time, the user should also sort and backup the relevant data frequently, in this way it can also be effective for avoiding problems of data difficult to restore caused by data loss. 
The second is to develop enterprise network security management technology under the cloud computing technology. In terms of the computer itself, the user must learn how to operate a firewall or other security measures. Introducing either differential authorization systems or professional firewalls actively can promote the effectiveness and security of enterprise network in specific application. The application of digital signature technology also can promote enterprise network security management system under the condition of cloud computing technology and make practical performance of this system get relatively good promotion in specific application. Therefore, only when after enterprise network security gets real security can a harmonious and orderly new network management situation be formed to promote the development of the all enterprises' network security management system and give cloud users safer, more comfortable, high-quality service. On this basis, it should also constantly improve the process of the development and application of enterprise new network security technology, constantly promoting unfailing performance for enterprise network management system to deal with all kinds of threats under the condition of cloud computing.

The third is that it must control the security threats enterprise network security program faced with more strictly under the condition of cloud computing. In order to ensure the security of enterprise network program, it should use the means including intercepting unsanctioned information and data at any time etc. to prevent the constant intrusion of all kinds of outside information and data and have reasonable protection during the installation of computer network protection program. The server under the cloud computing technology can be hidden in the inner network of the enterprise, in this way it can save the public network IP and do real time monitoring on each visit website and thus promote the security degree of enterprise network system under the condition of cloud computing. The enterprise cloud computing serves can also use level-to-level administration so as to avoid data leakage of enterprise customers to a great extent. Besides, the level-to-level administration can make mamagement of each level get monitored. In this, data under the condition of cloud computing can be managed by a lot of people. Generally speaking, the duties of workers for operation and maintenance are mainly handling the work on aspects of operation and maintenance well. The workers with core permissions though can get to relevant information of enterprise users, they can not delete relevant information of enterprise users randomly because they will be restricted by the relevant process of operation and maintenance. In this way, it can promote the security level of enterprise network program very well.

\section{Conclusion}

To sum up, with the constant development of modern network technology, based on cloud computing technology, the construction of enterprise network security system has become an important problem modern people concern. The relevant parties shall make their own efforts on how to solve this complex problem. For the general business users, they must have a strong sense of responsibility of network security; For cloud computing operating companies, they must practically promote technical development strength, improving the safety performance of enterprise network management system on the maximum; For the national legislature, they must work out as soon as possible to the legal norms to better maintain the security of network system and form an internet security management system under cloud computing technology and create more secure and efficient modern enterprise network security management system.

\section{References}

[1] Yang Xi. Computer security problems and countermeasures under the background of cloud computing . Information security and technology, 2013 (8).

[2] Ji Ling, Tan Liang. Computer security problems and coping methods analysis under the backgroung of cloud computing. Electronic technology and software engineering, 2014 (3). 
[3] Mao Lihua. Network security technical implementation path analysis under the cloud computing.Network security technology and application, 2014 (6).

[4] Na Yong. Computer network security strategy research under the environment of cloud computing. Electronic production, 2014 (10).

[5] Jing Yi Qing. The network security problems and countermeasures study under cloud computing environment. Network security technology and application, 2015 (9).

[6] Yong-hua Luo, Qiu Shang Ming, Yao Youmin. Computer security problems and countermeasures under the background of cloud computing. Electronic production, 2015 (14). 